\title{
Kinetic and MD modelling of automotive fuel droplet heating and evaporation: recent results
}

\author{
S.S. Sazhin ${ }^{1, *}$, I.N. Shishkova ${ }^{2}$ \\ ${ }^{1}$ Sir Harry Ricardo Laboratories, Advanced Engineering Centre, School of Computing, \\ Engineering and Mathematics, University of Brighton, Brighton BN2 4GJ, UK \\ ${ }^{2}$ Low Temperature Department, Moscow Power Engineering Institute, Krasnokazarmennaya \\ 14, Moscow 111250, Russia \\ *Corresponding author: S.Sazhin@brighton.ac.uk
}

\begin{abstract}
Recent results of the investigation of kinetic and molecular dynamics (MD) models for automotive fuel droplet heating and evaporation are summarised. The kinetic model is based on the consideration of the kinetic region in the close vicinity of the surface of the heated and evaporating droplets, where the motion of molecules is described in terms of the Boltzmann equations for vapour components and air, and the hydrodynamic region away from this surface. The effects of finite thermal conductivity and species diffusivity inside the droplets and inelastic collisions in the kinetic region are taken into account. A new self-consistent kinetic model for heating and evaporation of Diesel fuel droplets is briefly described. The values of temperature and vapour densities at the outer boundary of the kinetic region are inferred from the requirement that both heat flux and mass flux of vapour components in the kinetic and hydrodynamic regions in the vicinity of the interface between these regions are equal. At first, the heat and mass fluxes in the hydrodynamic region are calculated based on the values of temperature and vapour density at the surface of the droplet. Then the values of temperature and vapour density at the outer boundary of the kinetic region, obtained following this procedure, are used to calculate the corrected values of hydrodynamic heat and species mass fluxes. The latter in their turn lead to new corrected values of temperature and vapour density at the outer boundary of the kinetic region. It is shown that this process quickly converges and leads to self-consistent values for both heat and mass fluxes. Boundary conditions at the surface of the droplet for kinetic calculations are inferred from the MD calculations. These calculations are based on the observation that methyl $\left(\mathrm{CH}_{3}\right)$ or methylene $\left(\mathrm{CH}_{2}\right)$ groups in n-dodecane (approximation of Diesel fuel) molecules can be regarded as separate atom-like structures in a relatively simple United Atom Model. Some results of the application of quantum chemical methods to the estimation of the evaporation/condensation coefficient are discussed.
\end{abstract}

\section{Keywords}

Diesel fuel, droplets, heating, evaporation, kinetic modelling.

\section{Introduction}

In most applications, including those focused on automotive fuel droplets, the modelling of heating and evaporation processes has been based on the assumption that vapour at the liquid surface is saturated and the problem of evaporation reduces to the analysis of diffusion of vapour from the liquid (droplet) surface to the ambient gas [1]. The limitations of this approximation have been well known for over a century (see the references in [2]). In a number of studies, summarised in [1], the evaporation of n-dodecane $\mathrm{C}_{12} \mathrm{H}_{26}$ or a mixture of $n$-dodecane and $p$ dipropylbenzene $\mathrm{C}_{12} \mathrm{H}_{18}$ (approximating alkanes and aromatics in Diesel fuel) was studied and a new model based on a combination of the kinetic and hydrodynamic approaches was developed. In the close vicinity of droplet surfaces (up to one hundred molecular mean free paths), the vapour and ambient gas dynamics were studied based on the kinetic Boltzmann equations for vapour and air (kinetic region), while at larger distances the analysis was based on the conventional hydrodynamic equations (hydrodynamic region). These regions alongside the liquid region are schematically shown in Figure 1. Mass fluxes of components, momentum and energy fluxes were conserved at the interface between kinetic and hydrodynamic regions. The boundary conditions at the droplet surface were specified in terms of the evaporation coefficient which was estimated using molecular dynamics (MD) or quantum chemical (QC) methods.

The purpose of this paper is to provide an overview of the most important results in kinetic, MD and QC analysis of automotive fuel droplet heating and evaporation, mainly obtained since the publication of [1]. This overview is complementary to the more general review [3] recently published in Fuel.

The fundamentals of the most advanced self-consistent kinetic model for automotive fuel droplet heating and evaporation are summarised in Section 2, following [4]. Basic principles of the United Atom Model, used for the MD estimation of the evaporation coefficient (used as the boundary condition at the surface of the droplet in kinetic modelling), are briefly described in Section 3 , following $[5,6]$. The feasibility of using quantum chemical methods for studies of the processes at the surface of droplets and estimation of the evaporation coefficient is discussed in Section 4, following [7]. The main results of the paper are summarised in Section 5. 


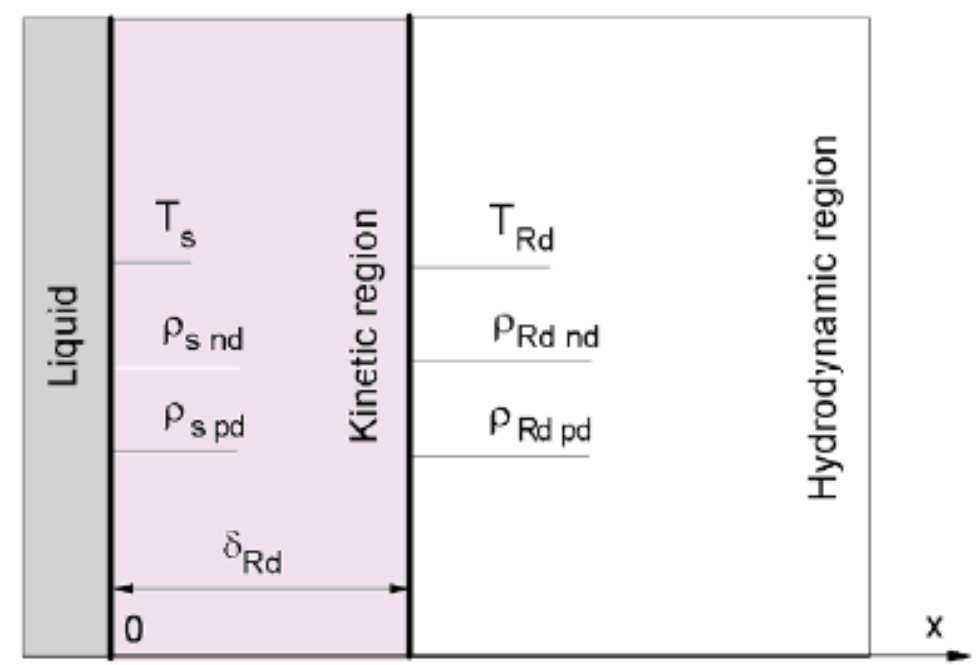

Figure 1. Liquid, kinetic and hydrodynamic regions near the surface of the droplet. $T_{s}$ is the droplet surface temperature, $\rho_{s(n d, p d)}$ are n-dodecane $\left(_{n d}\right)$ and p-dipropylbenzene $\left(_{p d}\right)$ vapour densities in the immediate vicinity of the droplet surface, $T_{R d}$ and $\rho_{R d(n d, p d)}$ are the temperature and $\mathrm{n}$-dodecane $\left({ }_{n d}\right)$ and $\mathrm{p}$-dipropylbenzene $\left({ }_{p d}\right)$ vapour densities at the outer boundary of the kinetic region. Reprinted from International Journal of Heat and Mass Transfer, Volume 93, Sazhin et al., A self-consistent kinetic model for droplet heating and evaporation, Pages 1206-1217, Copyright Elsevier (2016).

\section{A self-consistent kinetic model}

As already mentioned, the boundary condition at the interface between the kinetic and hydrodynamic regions has been inferred from the requirement of the conservation of heat and mass (or species mass) fluxes at this interface. In all papers published before [4], hydrodynamic heat and mass fluxes were calculated based on the simplifying assumptions that the temperature at the outer boundary of the kinetic region is the same as the droplet surface temperature and vapour pressure at this boundary is the same as the saturated vapour pressure at the droplet surface. This approach allows one to find the corrected values of temperature and vapour density at the interface between the kinetic and hydrodynamic regions.

The main problem with this approach is that the heat and mass (or species mass in the case of multi-component droplets) fluxes in the hydrodynamic region, calculated based on these corrected values of temperature and vapour density, are not necessarily equal to the heat and mass fluxes in the hydrodynamic region used to find these corrected values. The authors of [4] suggested that this problem can be overcome if an iterative process is used. In this case, the corrected values of temperature and vapour density (or species densities) at the outer boundary of the kinetic region were used to calculate the corrected values of hydrodynamic heat and mass fluxes. The latter leads to new corrected values of temperature and vapour density at the outer boundary of the kinetic region. If this process converges then self-consistent values for both heat and mass fluxes are obtained.

This iterative procedure is illustrated in Figures 2 and 3 in which the process of calculation of density and temperature at the interface between the kinetic and hydrodynamic regions is shown. Diesel fuel is approximated by $\mathrm{n}$-dodecane $\left(\mathrm{C}_{12} \mathrm{H}_{26}\right)$, a droplet is assumed to be moving with constant relative velocity equal to $10 \mathrm{~m} / \mathrm{s}$ and its surface temperature is equal to $600 \mathrm{~K}$; gas temperature and pressure are taken equal to $1000 \mathrm{~K}$ and 30 bar respectively. The calculations are based on the Effective Thermal Conductivity (ETC) model (see [1] for the details). The plots of $\tilde{j}_{\mathrm{k}} \equiv j_{\mathrm{kn}} /\left(\rho_{0} \sqrt{R_{v} T_{0}}\right)$ versus $\alpha_{\rho}$ and $\tilde{q}_{\mathrm{k}} \equiv q_{\mathrm{k}} /\left(p_{0} \sqrt{R_{v} T_{0}}\right)$ versus $\alpha_{T}$ are shown in these figures (lines ' $\mathrm{k}$ '). Also, the plots of $\tilde{j}_{\mathrm{h}} \equiv j_{\mathrm{h} \mathrm{n}} /\left(\rho_{0} \sqrt{R_{v} T_{0}}\right)$ versus $\alpha_{\rho}$ and $\tilde{q}_{\mathrm{h}} \equiv q_{\mathrm{h}} /\left(p_{0} \sqrt{R_{v} T_{0}}\right)$ versus $\alpha_{T}$ (assuming that $\alpha_{\rho} \equiv \rho_{R d} / \rho_{s}=1$ and $\alpha_{T} \equiv T_{R d} / T_{s}=1$ ) are shown in the same figures (lines ' $h$ ', iteration 1). The intersection between these two pairs of lines gives the updated values $\alpha_{\rho}=0.979$ and $\alpha_{T}=1.031$.

In the kinetic models used before [4], these corrections were directly used for calculation of mass and heat fluxes (see [1] for the details). In the new model these corrections are used to update the values of $\tilde{j}_{\mathrm{h}}$ and $\tilde{q}_{\mathrm{h}}$. The updated values of these fluxes are shown in Figures 2 and 3 (lines 'h', iteration 2). The intersections of these lines with lines $\tilde{j}_{\mathrm{k}}$ and $\tilde{q}_{\mathrm{k}}$ give us new updated values $\alpha_{\rho}=0.969$ and $\alpha_{T}=1.029$. The following iterations, up to iteration 50 , lead to relatively small changes in these corrections, as shown in Figures 2 and 3 . The difference between the values of $\alpha_{\rho}$ and $\alpha_{T}$ decreases with increasing iteration number; the differences between the values inferred from the 49th iteration are almost indistinguishable from those inferred from the 50th iteration. Note that, in contrast to the previously used non-self-consistent models, the approach described above does not rely on the observation that $\tilde{q}_{\mathrm{k}}$ is almost independent of $\alpha_{\rho}$ and $\tilde{j}_{\mathrm{k}}$ is almost independent of $\alpha_{T}$.

The analysis as presented in Figures 2 and 3 was repeated for other droplet surface temperatures in the range $300-650 \mathrm{~K}$ and gas temperatures $800 \mathrm{~K}, 1000 \mathrm{~K}$ and $1200 \mathrm{~K}$. The same analysis was performed for bi-component droplets $(80 \% \mathrm{n}$-dodecane and $20 \% \mathrm{p}$-dipropylbenzene mixture) for gas temperature equal to $1000 \mathrm{~K}$.

The new model was applied to the analysis of heating and evaporation of $n$-dodecane droplets with initial radii and 


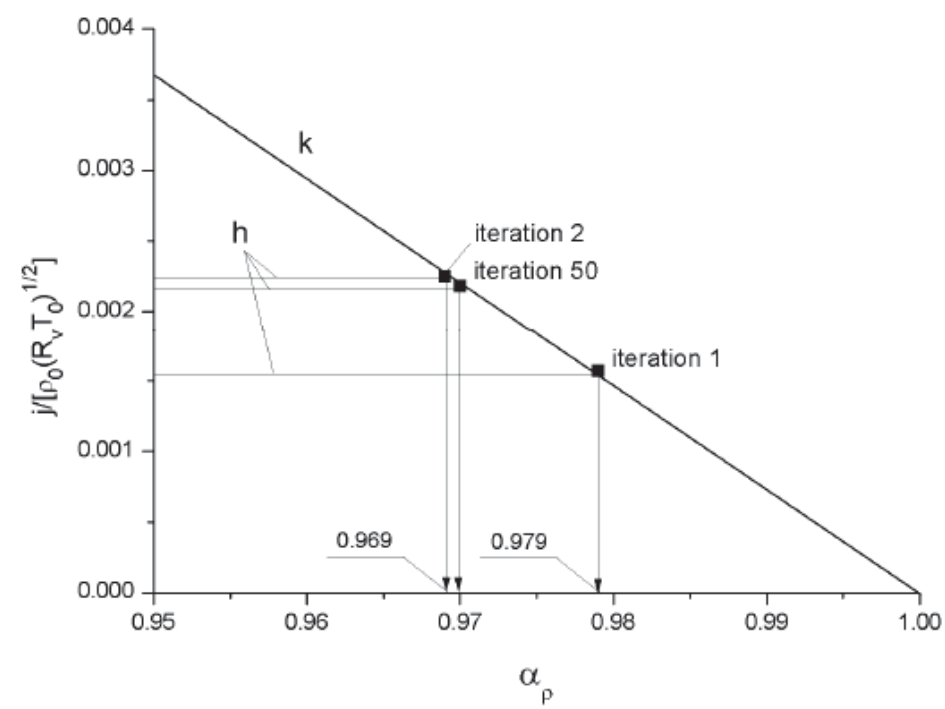

Figure 2. The plots of normalised mass fluxes $\tilde{j} \equiv j /\left(\rho_{0} \sqrt{R_{v} T_{0}}\right)$ predicted by the kinetic (line 'k') and hydrodynamic (lines ' $\mathrm{h}$ ') models for an n-dodecane droplet moving with constant velocity $10 \mathrm{~m} / \mathrm{s}$ versus $\alpha_{\rho} \equiv \rho_{R d} / \rho_{s}$ (normalised vapour density at the outer boundary of the kinetic region). Droplet surface and gas temperatures are assumed equal to $600 \mathrm{~K}$ and $1000 \mathrm{~K}$ respectively. Reprinted from International Journal of Heat and Mass Transfer, Volume 93, Sazhin et al., A self-consistent kinetic model for droplet heating and evaporation, Pages 1206-1217, Copyright Elsevier (2016).

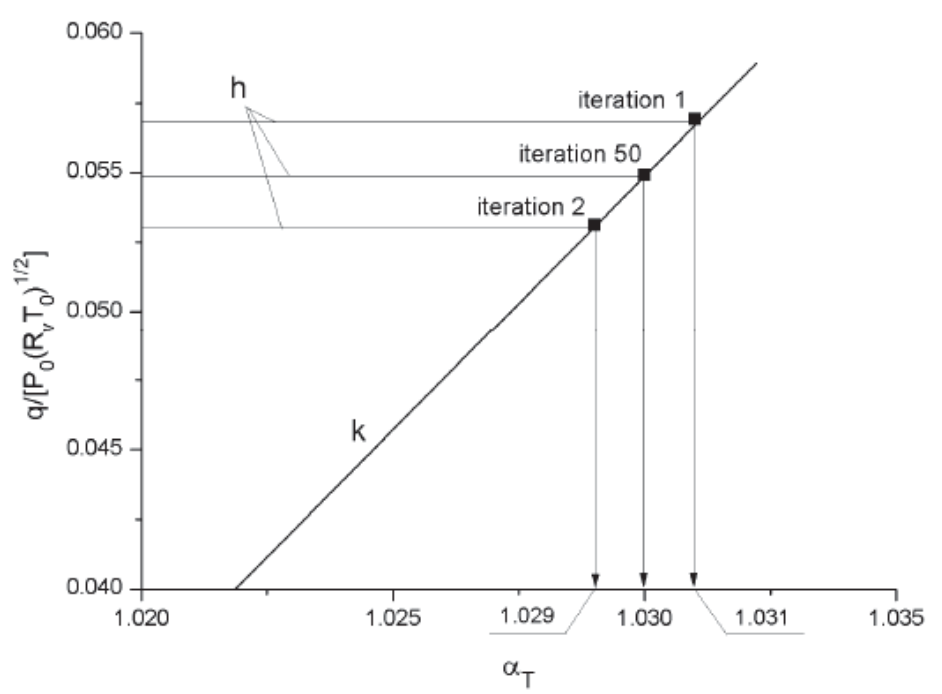

Figure 3. The plots of normalised heat fluxes $\tilde{q} \equiv q /\left(p_{0} \sqrt{R_{v} T_{0}}\right)$ predicted by the kinetic (line ' $\mathrm{k}$ ') and hydrodynamic (lines ' $\mathrm{h}$ ') models for an n-dodecane droplet moving with constant velocity $10 \mathrm{~m} / \mathrm{s}$ versus $\alpha_{T} \equiv T_{R d} / T_{s}$ (normalised temperature at the outer boundary of the kinetic region). Droplet surface and gas temperatures are assumed equal to $600 \mathrm{~K}$ and $1000 \mathrm{~K}$ respectively. Reprinted from International Journal of Heat and Mass Transfer, Volume 93, Sazhin et al., A self-consistent kinetic model for droplet heating and evaporation, Pages 1206-1217, Copyright Elsevier (2016). 
temperature equal to $5 \mu \mathrm{m}$ and $300 \mathrm{~K}$, immersed into gas with temperatures equal to $800 \mathrm{~K}, 1000 \mathrm{~K}$ and $1200 \mathrm{~K}$ and pressure equal to 30 bar. Droplets were either stationary or moving with constant velocity equal to $10 \mathrm{~m} / \mathrm{s}$. It was shown that in all cases the kinetic effects result in a decrease in droplet surface temperature and an increase in the evaporation time. This increase was shown to be particularly important for higher gas temperatures and moving droplets $[1,3,4]$. The contribution of p-dipropylbenzene was shown to decrease the kinetic effects on droplet evaporation time [4].

The analysis presented in this section is based on the assumption that droplets are perfect spheres. The kinetic model for non-spherical droplets has not yet been developed, to the best of our knowledge. The results of development of a hydrodynamic model of spheroidal droplet heating and evaporation are described in [8].

\section{United Atom Model}

As already mentioned, the solution to the Boltzmann equation in the kinetic region requires the formulation of the boundary condition at the liquid/gas interface. This boundary condition is essentially controlled by the evaporation coefficient (see review [9] for the details). A theoretical estimation of this coefficient requires the application of molecular dynamics (MD) methods [1]. A number of models have been developed to describe the molecular dynamics of complex hydrocarbon molecules, such as n-dodecane. Most of these models are based on the observation that the $\mathrm{C}-\mathrm{H}$ bond in complex hydrocarbon molecules is much shorter and much stronger than the $\mathrm{C}-\mathrm{C}$ bond, and also stronger than the van der Waals forces between molecules. Thus the methyl $\left(\mathrm{CH}_{3}\right)$ or methylene $\left(\mathrm{CH}_{2}\right)$ groups in the n-dodecane molecule can be regarded as separate atom-like structures in a relatively simple United Atom Model. In [5, 6] this model was used for the estimation of the evaporation coefficient at the surface of $n$-dodecane droplets based on MD calculations.

The contributions of the Lennard-Jones, bond bending and torsion potentials with the bond length constrained were taken into account. The analysis of [5] was based on 400 molecules, while the analysis of [6] was based on 720 molecules. The results of MD calculations of the evaporation coefficient $\beta$, which are presented in the latter paper, were approximated by the following simple formula (see [1] for the details):

$$
\beta\left(T_{s}\right)=7 \times 10^{-6} T_{s}^{2}-9.8 \times 10^{-3} T_{s}+3.7215,
$$

where $T_{s}$ is the droplet surface temperature (in $\mathrm{K}$ ). The values of $\beta$ predicted by Expression (1) turned out to be compatible with results reported by a number of other authors (see Figure 4).

Note that the United Atom Model is not valid at temperatures close to the critical temperature. In this case, a much more complex All Atom Model, taking into account the contributions of all atoms should be used. Also, the United Atom Model does not explicitly take into account quantum chemical effects. The importance of these effects is discussed in the next section.

\section{Quantum chemical effects}

The analysis summarised in the previous section did not explicitly take into account quantum chemical (mechanical) effects when analysing molecular dynamics in the vicinity of the droplet surface. In a series of our previous papers, the results of which are reviewed in [7, 3], the applicability of this approach has been investigated. In this section, the main findings of this investigation are summarised.

\section{Quantum chemical methods}

Although the solution to the Schrödinger equation for the wave function $\psi$ in some simple cases (e.g. an isolated hydrogen atom) is well known, its general solution in the case when many atoms need to be analysed simultaneously is still a challenge for quantum mechanical modelling. Perhaps the most widely used simplified method for solving this equation is the Hartree-Fock (HF) method. Two strategies for application of the HF method for practical calculations have been suggested. In the semi-empirical methods the integrals used in the HF method are estimated based on experimental data or based on a series of rules which allow one to set certain integrals to zero. In the $a b$ initio methods an attempt is made to calculate all these integrals.

Although this method is widely used in practical computations, it is still an approximate one and demands considerable computational effort. This stimulated the development of alternative approaches to the calculation of electronic systems, including the Density Functional Theory (DFT) which is focused on the electron density $\left(\rho_{e}\right)$ rather than on the wave function $\psi$. In this approach it is assumed that the energy of a molecule is a function of the electron density. Since the electron density is a function of position $\rho_{e}(\mathbf{r})$, this energy appears to be a function of a function (functional) of density

On some occasions various approximations of the energy functional in the DFT, that incorporate parts of the exact exchange from the HF theory, have been used. One such approach is known as B3LYP (Becke, 3-parameter, Lee-Yang-Parr). Various semi-empirical quantum chemistry methods are important for dealing with large molecules where the HF method without the approximations (ab initio approach) and DFT are too expensive. In these cases a range of fitting parameters are typically used to produce the results. The parameters in the PM7 method were calibrated to obtain results consistent with experimental and ab initio data for more than 9000 compounds. The accuracy of the PM7 method is close to that of the $a b$ initio and DFT methods used with the 6-31G(d) basis set.

The fundamental differences between the classical molecular mechanics/molecular dynamics (MM/MD), semiempirical PM7, ab initio and DFT methods are in the way in which the contributions of electrons are taken into account. The contribution of all electrons is taken into account in ab initio and DFT with a self-consistent field 
(SCF); only valence electrons are considered in semi-empirical quantum-chemical methods (QCMs) with SCF, and no electrons are considered in classical MM/MD methods without SCF. A new continuum solvation model based on the quantum mechanical charge density of a solute molecule interacting with a continuum description of a solvent is known as the SMD. The term continuum indicates that the solvent is represented as a dielectric medium with surface tension at the solute-solvent boundary.

In the case of modelling of the transient processes, the Dynamic Reaction Coordinates (DRC) method has been widely used. The key concept of this method is the Dynamic Reaction Coordinate which is the path followed by all the atoms in a system assuming the conservation of energy. In contrast to conventional molecular dynamic (MD) approaches, in this approach the contributions of the processes at the electronic level are taken into account. These models have been implemented in a number of known programs, including Gaussian 09, WinGAMESS 2013 R1, and MOPAC2012.

\section{Interaction between molecules and droplets}

In this section, the results of the analysis of the collision processes between n-dodecane molecules and clusters/nanodroplets, based on the Dynamic Reaction Coordinate (DRC) method, are briefly summarised, following $[7,10]$. In the DRC approach, the total kinetic energy includes the kinetic energy of random thermal bond vibrations and rotations and the kinetic energy of the translational motion of the whole molecule. In our previous analysis, this approach was applied to study the dependence of sticking/scattering of $n$-dodecane molecules on their angles of attack, temperature, and cluster/nanodroplet size. The calculations were performed for molecules interacting with a cluster (7 molecules) or a nanodroplet ( 64 or 128 molecules) of $n$-dodecane molecules.

It was shown that at large angles of attack, molecules are absorbed by a cluster or nanodroplet of relatively small size (diameters in the range 2-7 nm) if the kinetic energy of attacking molecules is low and they are not oriented exactly towards one of the surface molecules. At angles close to $\approx 1^{\circ}$ almost perfectly elastic collisions were observed if the molecules had high kinetic energy ( $\sim 10 \mathrm{~kJ} / \mathrm{mol}$ or larger) and were oriented directly towards one of the surface molecules. It was assumed that the kinetic energy of the molecules in the clusters or nanodroplets was low and thermal vibrations and bond rotations corresponded to $300-400 \mathrm{~K}$. The kinetic energy of the attacking molecule was high with their effective temperatures being in the range 500-1200 K.

It was concluded that the probability of the attacking molecule sticking to a droplet is maximal if the molecular plane is parallel or almost parallel to the droplet surface (multi-point interactions of long $n$-dodecane molecules with the droplet surfaces are expected). If the kinetic energy of the attacking molecules is greater than that of boiling temperature then it is expected that their scattering and removal from the cluster/nanodroplet surface will take place. It was shown that molecule-nanodroplet interaction results depend on the kinetic energy and orientations of the attacking and surface molecules. The mechanisms of evaporation of microdroplets and nanodroplets were shown to involve different processes. In the case of microdroplets, individual carbon molecules were evaporated from their surfaces, while nanodroplets disintegrated into clusters and individual molecules.

The predicted decrease in the lifetime of the "surface" molecules with temperature when the temperature of nanodroplets increases agrees with the prediction of the classical theory based on MD simulations of $n$-dodecane molecules using the United Atom Model (see Equation (1)). The analysis described above, however, does not allow us to predict the evaporation coefficient, as we did in the MD analysis (see Equation (1)). To obtain the values of this coefficient, using this analysis, one would need to repeat these calculations for a wide range of angles of attack, orientation of molecules and energies for various conformers and various conditions of clusters and nanodroplets. Since this is not currently feasible, an alternative approach to calculating this coefficient, taking into account quantum chemical effects, is described in the next section, following $[11,7]$.

\section{Evaporation/condensation coefficient}

In the analysis originally presented in [11] and summarised in [7] the transition state theory (TST) was used. This theory was based on a QC DFT approach taking into account the conformerisation of n-dodecane molecules (approximation of Diesel fuel). It was shown that the most accurate expression for the condensation coefficient (assumed to be equal to the evaporation coefficient) is the one averaged over the states of various conformers transferred between two phases. This was given by the following formula [11]:

$$
\left\langle\beta_{V}\right\rangle=\left\{1-\left[\frac{\rho_{g}}{\rho_{l}} \exp \frac{\left\langle\Delta G_{g \rightarrow l}\right\rangle}{R_{u} T}\right]^{1 / 3}\right\} \exp \left\{-0.5\left[\left[\frac{\rho_{g}}{\rho_{l}} \exp \frac{\left\langle\Delta G_{g \rightarrow l}\right\rangle}{R_{u} T}\right]^{1 / 3}-1\right]^{-1}\right\},
$$

where $R_{u}$ is the universal gas constant, $\rho_{g(l)}$ is the gas (liquid) density, $\Delta G_{g \rightarrow l}$ is the change in the Gibbs free energy during the condensation process, subscript ${ }_{V}$ shows that the expression $\beta_{V}$ depends on specific volumes, \langle\rangle shows the averaging over the states of various conformers transferred between two phases. The effects of both the conformerisation and cross-conformerisation of molecules were taken into account. Ninety-five stable conformers were selected based on the changes in the Gibbs free energy.

A comparison between the results of calculations of $\beta=\beta_{V}$ based on Expression (2) and those obtained previously is shown in Figure 4, reproduced from [7]. As can be seen from this figure, taking into account QC effects leads to marginal modification of the predicted evaporation/condensation coefficient unless the temperature approaches the critical temperature. Thus, although the analysis of QC effects takes into account many new effects ignored in the conventional force fields (FF) approach, the contribution of these effects to the values of the evaporation coefficient turned out to be marginal. 


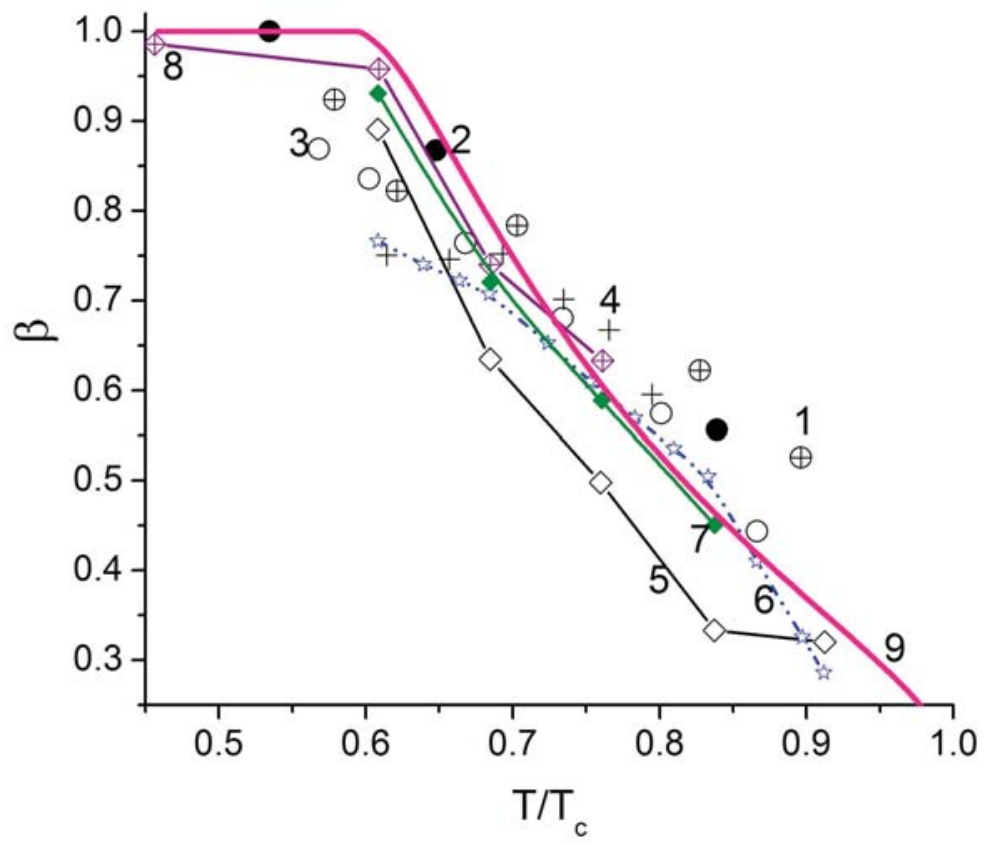

Figure 4. Comparison of the values of the evaporation coefficient $\beta$, predicted by MD, FF (symbols 1-4, curves 5-8) and Expression (2) (curve 9), versus normalised temperature $\left(T / T_{c}\right.$ ( $T_{c}$ is the critical temperature)). Symbols (1-4) refer to the models for structureless LJ fluids with various input parameters (see [7] for the details), curves 5 and 7 refer to the results obtained from the United Atom Model, curve 6 refers to the results of calculations based on the TST model reproduced from [5], curve 8 is based on the results of calculations using the model described by Mizuguchi et al. [12]. QC calculations were performed using DFT $\omega$ B97X-D/cc-pVTZ and SMD/ $\omega$ B97X-D/cc-pVTZ. Reprinted from Fuel, Volume 165, Sazhin et al., Quantum-chemical analysis of the processes at the surfaces of Diesel fuel droplets, Pages 405-412, Copyright Elsevier (2016).

\section{Conclusions}

The results of several recent developments of kinetic and molecular dynamics (MD) models for automotive fuel droplet heating and evaporation are described, based on previously published journal papers (mainly from 20152017). The kinetic models, developed in a series of our recent papers, are based on consideration of the kinetic region in the immediate vicinity of the surface of the heated and evaporating droplets and the hydrodynamic region away from this surface. In the kinetic region the motion of molecules was described in terms of the Boltzmann equations for vapour components and air. The effects of finite thermal conductivity, recirculation and species diffusivity inside the liquid droplets were taken into account, alongside the effects of inelastic collisions in the kinetic region. A self-consistent kinetic model for heating and evaporation of Diesel fuel (approximated by $n$-dodecane $\mathrm{C}_{12} \mathrm{H}_{26}$ or a mixture of n-dodecane and p-dipropylbenzene $\mathrm{C}_{12} \mathrm{H}_{18}$ (approximating alkanes and aromatics in Diesel fuel)) droplets is described. In this model, the values of temperature and vapour density at the outer boundary of the kinetic region are inferred from the requirement that the heat flux and mass flux of vapour components in the kinetic and hydrodynamic regions in the vicinity of the interface between these regions are equal. At first, the heat and mass fluxes in the hydrodynamic region were calculated based on the values of temperature and vapour density at the surface of the droplet. Then the values of temperature and vapour density at the outer boundary of the kinetic region, obtained following this procedure, were used to calculate the corrected values of hydrodynamic heat and species mass fluxes. The latter in their turn led to new corrected values of temperature and vapour density at the outer boundary of the kinetic region. It was shown that this process quickly converges and leads to self-consistent values for both heat and mass fluxes.

Boundary conditions at the surface of the droplet for kinetic calculations were formulated in terms of the evaporation coefficient, the values of which were inferred from the molecular dynamics (MD) calculations. These calculations were based on the United Atom Model. The latter model is based on the observation that methyl $\left(\mathrm{CH}_{3}\right)$ or methylene $\left(\mathrm{CH}_{2}\right)$ groups in $\mathrm{n}$-dodecane (approximation of Diesel fuel) molecules can be regarded as separate atom-like structures. Some recent results of the application of quantum chemical methods to the analysis of the interaction between molecules and the surfaces of molecular clusters and nanodroplets are summarised. It is shown that quantum-chemical (QC) analysis leads to marginal modifications of the predicted evaporation coefficient, using the United Atom Model, unless the temperature approaches to the critical temperature for $\mathrm{n}$-dodecane.

\section{Acknowledgements}

The authors are grateful to the EPSRC (UK) (grants EP/K005758/1, EP/K020528/1 and EP/M002608/1), the Royal Society (UK) (grant IE 160014) and the Russian Foundation for Basic Research (grant No. 17-08-01274) for their financial support. 


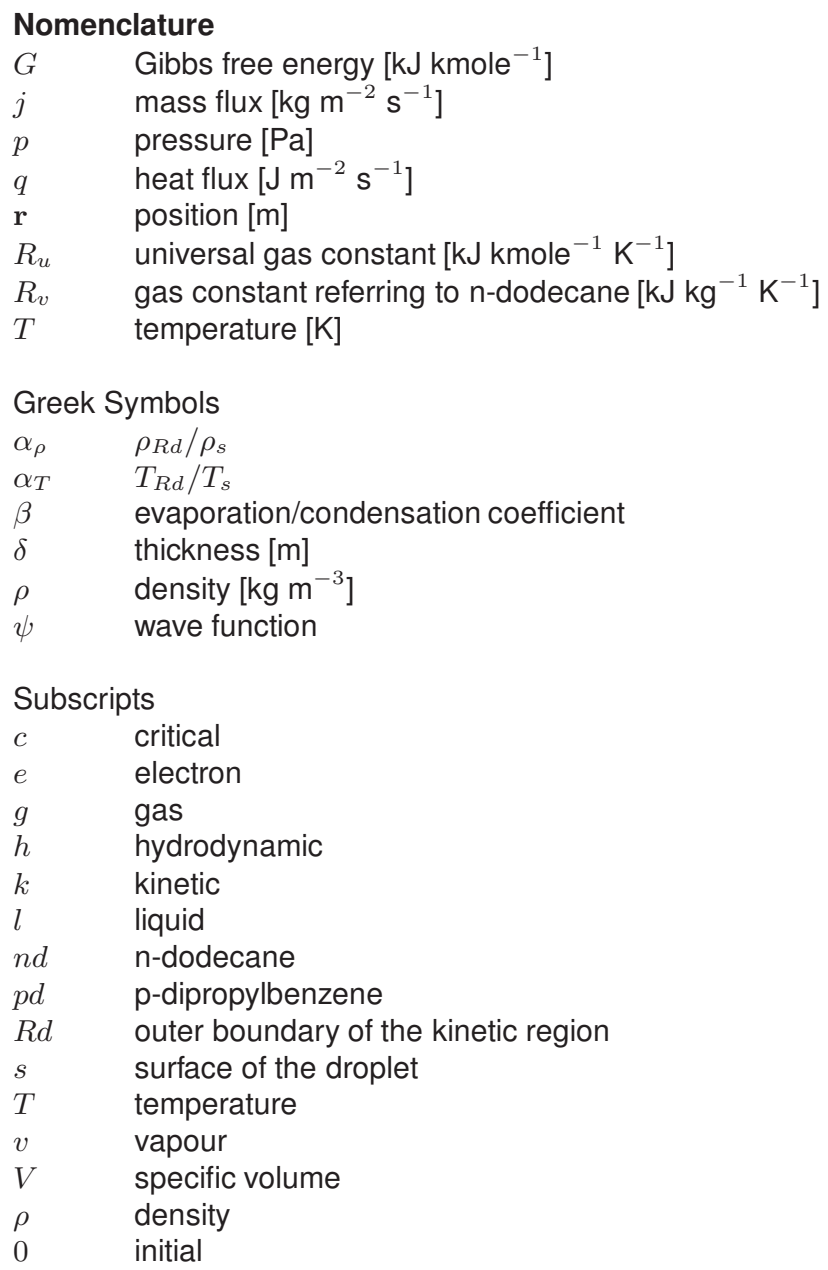

\section{References}

[1] Sazhin, S.S., 2014, "Droplets and Sprays". Springer.

[2] Fuchs, N.A., 1959, "Evaporation and Droplet Growth in Gaseous Media". London: Pergamon Press.

[3] Sazhin, S.S., 2017, Fuel, 196, pp. 69-101.

[4] Sazhin, S.S., Shishkova, I.N., Al Qubeissi, M., 2016, International Journal of Heat and Mass Transfer, 93, pp. 1206-1217.

[5] Cao, B.-Y., Xie, J.-F., Sazhin, S.S., 2011, Journal of Chemical Physics, 134, 164309.

[6] Xie, J.-F., Sazhin, S.S., Cao, B.-Y., 2011, Physics of Fluids, 23 (11), 112104.

[7] Sazhin, S.S., Gun'ko, V.M., Nasiri, R., 2016, Fuel, 165, pp. 405-412.

[8] Zubkov, V.S., Cossali, G.E., Tonini, S., Rybdylova, O., Crua, C., Heikal, M. and Sazhin, S.S., 2017, International Journal of Heat and Mass Transfer, 108, pp. 2181-2190.

[9] Persad, A.H., Ward, C.A., 2016, Chemical Reviews, 116 (14), pp. 7727-7767.

[10] Gun'ko, V.M., Nasiri, R., Sazhin, S.S., 2015, Fluid Phase Equilibria, 366, pp. 99-107.

[11] Gun'ko, V.M., Nasiri, R., Sazhin, S.S., 2015, Journal of Chemical Physics, 142 (3), 034502.

[12] Mizuguchi, H., Nagayama, G., Tsuruta, T., 2010, Seventh International Conference on Flow Dynamics, November 1st-3rd, 2010, Sendai International Center, p. 386. 